\title{
Article
}

\section{First-Principles Study on Redox Magnetism and Electrochromism of Cyclometalated Triarylamine-Core Triruthenium Complex}

\author{
Lin Li ${ }^{1}$, Yue Wang ${ }^{1}$, Meng-Yang Chen ${ }^{1}$, Jian Zhang ${ }^{1}$, Jian-Quan Liang ${ }^{1}$, He-Qian Liu ${ }^{1}$, Yu-Xuan Sun ${ }^{2}$, \\ Wei Song ${ }^{2, *}$ and Wei-Feng Sun ${ }^{2, *}$
}

1 Electric Power Research Institute, State Grid Heilongjiang Electric Power Co. Ltd., Harbin 150040, China; lil@hepri.hl.sgcc.com.cn (L.L.); wangy@hepri.hl.sgcc.com.cn (Y.W.); chenmy@hl.sgcc.com.cn (M.-Y.C.); zhangj@hepri.hl.sgcc.com.cn (J.Z.); liangjq@hepri.hl.sgcc.com.cn (J.-Q.L.); liuhq@hepri.hl.sgcc.com.cn (H.-Q.L.)

2 Key Laboratory of Engineering Dielectrics and Its Application, Ministry of Education, School of Electrical and Electronic Engineering, Harbin University of Science and Technology, Harbin 150080, China; cosine_sun@163.com

* Correspondence: songwei@hrbust.edu.cn (W.S.); sunweifeng@hrbust.edu.cn (W.-F.S.); Tel.: +86-158-4659-2798 (W.-F.S.)

check for updates

Citation: Li, L.; Wang, Y.; Chen, M.-Y.; Zhang, J.; Liang, J.-Q.; Liu, H.-Q.; Sun, Y.-X.; Song, W.; Sun, W.-F. First-Principles Study on Redox Magnetism and Electrochromism of Cyclometalated Triarylamine-Core Triruthenium Complex. Crystals 2021, 11, 57. https://doi.org/10.3390/ cryst11010057

Received: 3 December 2020 Accepted: 11 January 2021 Published: 13 January 2021

Publisher's Note: MDPI stays neutral with regard to jurisdictional clai$\mathrm{ms}$ in published maps and institutional affiliations

Copyright: (C) 2021 by the authors. Licensee MDPI, Basel, Switzerland. This article is an open access article distributed under the terms and conditions of the Creative Commons Attribution (CC BY) license (https:// creativecommons.org/licenses/by/ $4.0 /)$.

\begin{abstract}
Spin electronic states and optical properties of a circular ruthenium $(\mathrm{Ru})$ terpyridine complex with a triarylamine core (CTTC) are theoretically investigated by first-principles calculations within an all-electron numerical orbital scheme based on spin density functional theory (SDFT), which demonstrate five well-defined redox states for electrochromic functions. Atomic structure of CTTC molecule is obtained by geometric optimization, and its electronic structure with a decreasing semiconductor band-gap exhibits five consecutive single-electron redox states of Ru-coordinated centers. Except for CTTC in $(\mathrm{Ru})_{3}+4$ redox state exhibiting a net spin of $2.25(\hbar / 2)$, the other redox states are almost zero in total spin. Density distribution and energy-splitting of spin states indicate that the ferromagnetic coupling of Ru cations coordinating with terpyridine/triarylamine ligands originates dominantly from the spin polarization of $\mathrm{Ru} 4 d$-orbitals coordinated by $\mathrm{N}$ - and $\mathrm{C}-2 p$ electrons of triarylamine. CTTC molecule in each redox state represents a well-discriminated absorption in visible region, with the highest characteristic peaks locating at 24.2, 20.2, 21.3, and 19.3/21.7 $\left(10^{3} \mathrm{~cm}^{-1}\right)$ and a manifold of peaks at 13.4 25.3 $\left(10^{3} \mathrm{~cm}^{-1}\right)$ for $+2 \sim+6$ redox states, respectively. Theoretical electronic structure and optics of CTTC complex are used to evaluate the underlying physical mechanism of realizing a multi-color visible electrochromism by four couples of redox pairs, which is suggested to be applied for monitoring electrical information.
\end{abstract}

Keywords: multistate redox; electrochromism; first-principles calculation; electronic state; optical absorption spectrum

\section{Introduction}

Electrochromism of electroactive molecules is a manifestation of the reversible switch between distinguishable redox states under electric excitation, displaying reversible alternations in optical properties and transparency, which plays an important role in energy conservation, building, and aerospace at present [1-3]. Electrochromic molecules with a specific multistate redox can present consecutively shifting optical signals under low electric excitation that could be precisely identified for monitoring characteristic electrical information and constructing multiple channel outputs of molecular switches [4-6]. Redox switching between three states has been comprehensively reported recently for electrochromics, while it is still a challenge for more than four redox states [7-10]. Since the higher and lower redox states can be reduced and oxidized respectively under ambient atmosphere and temperature, multistate redox reactions in specific functional molecules are 
required to occur at relatively low-oxidation states. Meanwhile, the adjacent redox states should evidently be distinguished to avert disproportionation reactions and realize welldefined optical responses for electrochromic applications. Furthermore, electrochromic molecular switches are primarily implemented on the surface of transparent electrodes by depositing individual molecules [11-13]. As a result, in practical applications, the multistate molecules are separated in space to fulfill self-charge transfer for redox reactions. Especially, the insufficient stability of transparent electrodes and solid-state electrolytes in a certain voltage window limits the full potential of electrochromic molecules experimentally discovered to date [14]. Therefore, it is pertinent to investigate electrochromic mechanisms by quantum mechanics calculations of individual molecules, which can essentially elucidate the electronic states and optoelectronic transitions for molecular designs.

Ruthenium-amine-conjugated (cyclometalated ruthenium) complexes with low potential $\mathrm{Ru}(\mathrm{III} / \mathrm{II})$-C bonds have recently been characterized to represent a sufficient absorption discrimination in visible and near-infrared (NIR) regions through redox processes of at least three oxidation states [15-18], which show potential to be efficiently applied for electrochromic switches and variable optical attenuators in fiber-optic communications [19-21]. Triggered by these cyclometalated ruthenium complexes with four redox states at lowpotentials, in the present study, we specifically investigate a cyclometalated triruthenium complex with a triarylamine core by first-principles calculations of electronic structure and optoelectronic transition in various redox states, which accounts for its magnetism and multistate-redox electrochromics.

\section{Theoretical Calculation Methodology}

A molecular model of the cyclometalated triruthenium complex with a triarylamine core and terpyridine terminals (CTTC) is initially constructed using Monte Carlo (MC) molecular simulation method [22] according to the reported chemical configuration, as shown in Figure 1 [23], which is used as the input structure for geometry optimization of energy minimization. Total energy, electron structure, and optical property of CTTC molecule are calculated using the first-principles method based on spin density functional theory (SDFT) as implemented in DMol3 program of Materials Studio v2017R2 (Accelrys Inc., San Diego, CA, USA) software package [24,25], which are performed utilizing all-electron numerical orbital basis-set scheme with PBEsol gradient-corrected (GGA) exchange-correlation functional [26]. Wave functions of intrinsic electron states are expended by double numerical polarized (DNP) basis-set with a global orbital cutoff of $5.0 \AA$ to adequately reduce the error from basis-set finiteness. Coordinating center $\mathrm{Ru}$ atoms with $4 d$ single-electron occupied states may cause spin-splitting and net spin, which is therefore incorporated by using different orbitals for the two spin states based on SDFT with Dirac relativistic quantum mechanical equations [27]. Interactions between electrons and atomic-core are described by all-electron-relativistic core treatment. Convergence toleration for self-consistent field (SCF) iteration is set as $1.0 \times 10^{-6} \mathrm{Ha} /$ atom $(1 \mathrm{Ha}=27.2 \mathrm{eV})$, and the direct-inversion iterative-subspace (DIIS) density mixing scheme with charge and spin mixing amplitudes of 0.2 and 0.5 respectively is used to relax electrons [28]. Thermal smearing of $0.002 \mathrm{Ha}$ orbital occupations is employed to expedite SCF convergence. Geometry optimization of total energy functional minimization is implemented with conjugate gradient algorithm to obtain convergence quality of energy, force, and displacement lower than $1.0 \times 10^{-5} \mathrm{Ha}, 0.002 \mathrm{Ha} / \AA$, and $0.005 \AA$, respectively [29]. Dispersion correction of exchange-correlation functional is included to evaluate weak dispersion interactions [30]. Non-polarized optical properties are calculated by time-dependent DFT (TD-DFT) excitation method with the adiabatic local exchange density-functional approximation (ALDA) kernel describing exchange-correlation terms [31].

CTTC is modeled as an isolated molecule in vacuum without consideration of its surrounding dielectric medium during calculations. The different redox states of CTTC model are distinguished by initially setting the total charge of $(\mathrm{Ru})_{3}$ (three $\mathrm{Ru}$ cations in union) in CTTC molecule to be $+2 \sim+6$ respectively, with the whole charge of CTTC 
molecule being balanced to zero, which is schemed as the starting point for SCF iteration to obtain the most nearby energetic minimum for electronic relaxations. By SCF iterations, different energy minimum points from the initial specifications on every redox state have been reached, implying that all these redox states really exist and are well modeled. On this ground, theoretical schemes for modeling different redox states of CTTC complex are feasible, which are hereby valid for multiple-energy-point calculations in geometry optimization to retain relaxed atomic structures of all the modeled redox states.

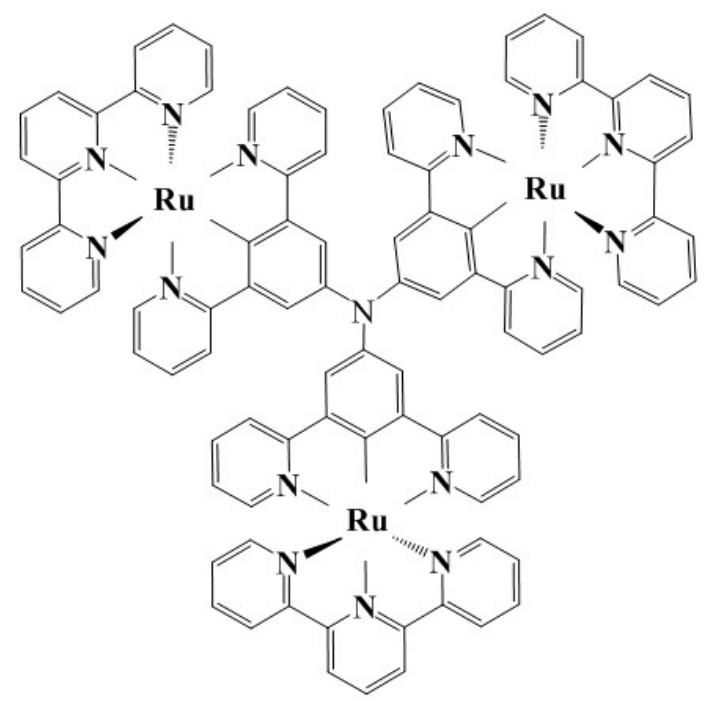

Figure 1. Schematic chemical configuration of triruthenium complex with a triarylamine core.

\section{Results and Discussion}

\subsection{Molecule Structure}

Although it is difficult to prepare CTTC molecular crystals in experiments due to the complex molecule structure, CTTC modeling has been accomplished on atomic scale to theoretically predict molecular structures by the energy-minimized geometry optimization in first-principles calculations, as representatively shown by the atomic-relaxed structures of +3 state CTTC molecule in Figure 2. According to geometric optimization, each redox state of CTTC complex is almost same in a stable coordination structure with a higher coordinating energy, as listed in Table 1 for the relaxed molecular structure of CTTC molecule in various redox states. CTTC molecule is in a pesudo- $\mathrm{C}_{3}$ symmetry around the central amine, with three $\mathrm{C}-\mathrm{N}$ bonds in a planar configuration and three $\mathrm{C}-\mathrm{N}-\mathrm{C}$ angles $\left(\angle \mathrm{CNC}\right.$ ) identically approaching $120^{\circ}$. Each unit phenyl of triarylamine core is in the same atomic plane with two pyridines bonding on both sides, which are perpendicular to the atomic plane of terpyridine terminal. In particular, three pyridine-phenyl-pyridine units of triarylamine core form a three-wheel propeller configuration with dihedral angles of $27.66^{\circ}$, $36.92^{\circ}$, and $43.54^{\circ}$, respectively, to the central amine plane. Cyclometalated ruthenium cations are sixfold coordinated by five pyridine $\mathrm{N}$ and one terminal $\mathrm{C}$ of triarylamine core in an octahedral configuration with a Ru-C bond length of $\sim 1.944 \AA$ and Ru-N coordinating lengths of 1.94 2.05 $\AA$. The averaged distance between ruthenium ions and the central nitrogen atom is around $6.18 \AA$. Furthermore, we have calculated the cohesive energy of a system constituting multiple CTTC molecules, demonstrating that van der Waals force between CTTC molecules is too weak to form molecular crystals, which is consistent with experimental reports [23]. 

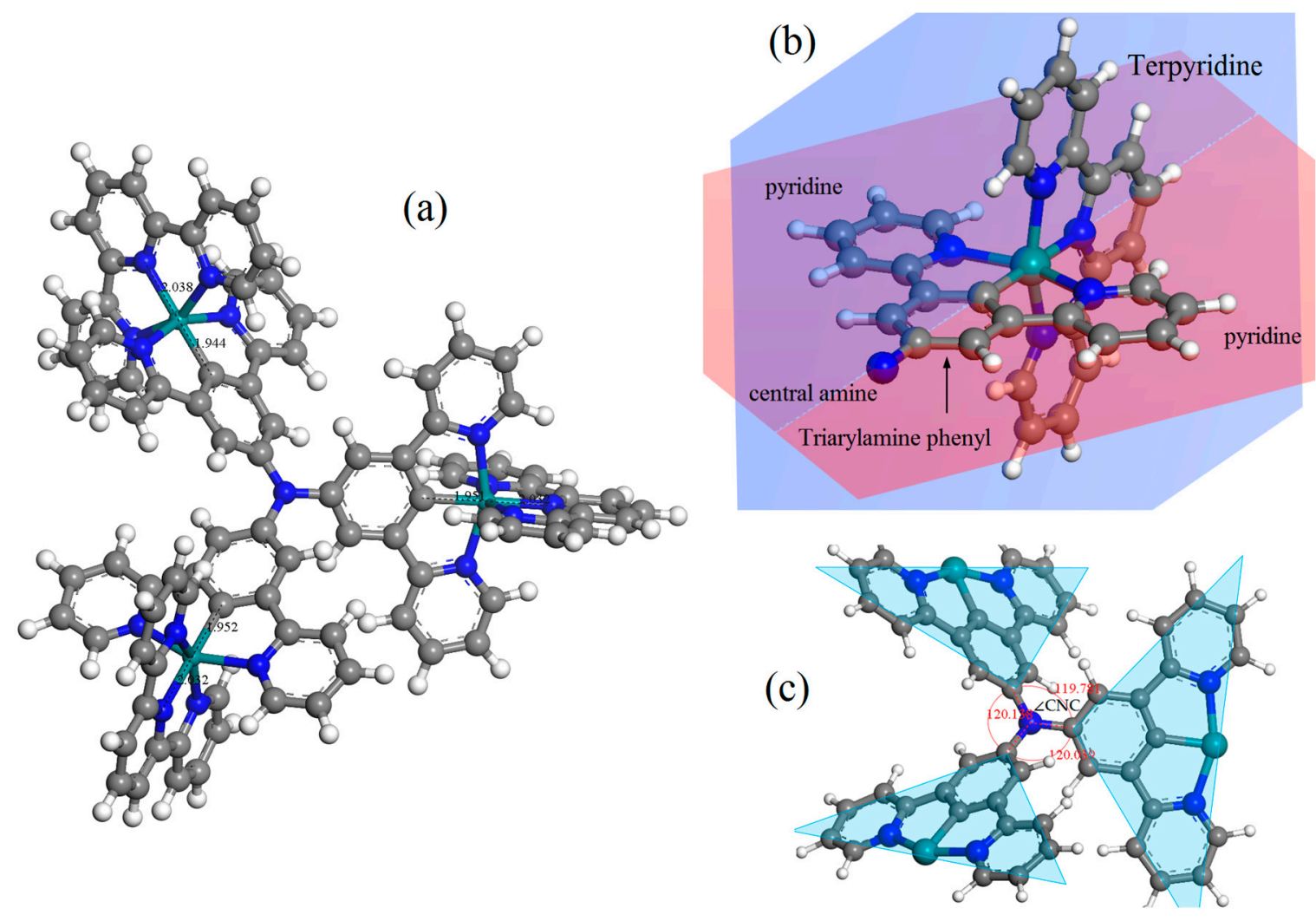

Figure 2. (a) Geometrically optimized molecular configuration and (b,c) the scaled local schematics showing atomic planes and bonding structures of CTTC molecule in +3 redox state as a representative.

Table 1. Molecular structures and energies of CTTC molecule in various redox states.

\begin{tabular}{|c|c|c|c|c|c|c|c|}
\hline \multirow{2}{*}{ Redox State } & \multicolumn{3}{|c|}{ Mean Bonding Length/Å } & \multirow{2}{*}{$\begin{array}{l}\text { Mean } \\
\angle \mathrm{CNCl}^{\circ}\end{array}$} & \multirow{2}{*}{$\begin{array}{c}\text { Triarylamine } \\
\text { Dihedral Angle/ }\end{array}$} & \multirow{2}{*}{$\begin{array}{c}\text { Total } \\
\text { Energy/Ha }\end{array}$} & \multirow{2}{*}{$\begin{array}{c}\text { Coordinating } \\
\text { Energy/Ha }\end{array}$} \\
\hline & $\mathrm{C}-\mathrm{N}$ & Ru-C & $\mathbf{R u}-\mathbf{N}$ & & & & \\
\hline+2 & 1.414 & 1.948 & 2.026 & 119.979 & $27.60 / 36.86 / 43.45$ & -17759.64085 & 1.4289 \\
\hline+3 & 1.416 & 1.944 & 2.034 & 119.986 & $27.66 / 36.92 / 43.54$ & -17759.64275 & 1.4276 \\
\hline+4 & 1.417 & 1.952 & 2.037 & 119.963 & $27.63 / 36.90 / 43.51$ & -17759.64295 & 1.4265 \\
\hline+5 & 1.419 & 1.949 & 2.025 & 119.998 & $27.54 / 36.68 / 43.36$ & -17759.64078 & 1.4296 \\
\hline+6 & 1.420 & 1.948 & 2.058 & 120.011 & $27.38 / 36.46 / 43.07$ & -17759.63343 & 1.4171 \\
\hline
\end{tabular}

\subsection{Multistate Redox Mechanism}

Ru complexes with a single metal-coordinated center will represent multistate redox reactions of coordination electron-pair offset caused by $\mathrm{Ru}(\mathrm{II}) / \mathrm{Ru}(\mathrm{III})$ transition under the electric field. Multiple coordination center atoms in polycore Ru complexes with the coordination bonds remaining during polymerization lead to multiple single-electron oxidation processes with the same oxidation potentials as their mono-core constitutes $[15,17]$. Therefore, a variety of consecutive reversible redox reactions can occur between the three $\mathrm{Ru}$ complexation centers and the $\mathrm{N}$ coordinating ligands of terpyridine and triarylamine, leading to the different coordination bonds $(\mathrm{Ru}-\mathrm{N})$ that well determine Ru-carbon (Ru-C) bonding characteristics, which may cause net spin for CTTC by spin polarization. We analyze the $\mathrm{Ru}-\mathrm{C}$ bonding and $\mathrm{Ru}-\mathrm{N}$ coordination properties by calculating deformation electron density to evaluate charge transfer between consecutive redox states of CTTC, as shown by SCF calculation results in Figure 3, in which deformation electron density is contoured on the atomic planes of terpyridine and triarylamine-phenyl, respectively. The maximum deformation electron density arises around $\mathrm{Ru}$ ions at the routes towards $\mathrm{C}$ and $\mathrm{N}$ atoms, implying that the orbital electrons of $\mathrm{Ru}$ atoms have been partially transferred 
from coordination centers to $\mathrm{N}$ and $\mathrm{C}$ atoms at octahedral vertices. Deformation density is illustrated by green-colored area around Ru cation, as shown in Figure 3, which is higher for +6 redox state than that for +3 state of CTTC molecule. It is hereby suggested that charge transfer from $\mathrm{Ru}$ to $\mathrm{C}$ and $\mathrm{N}$ will be exacerbated when CTTC alters towards a higher redox state.

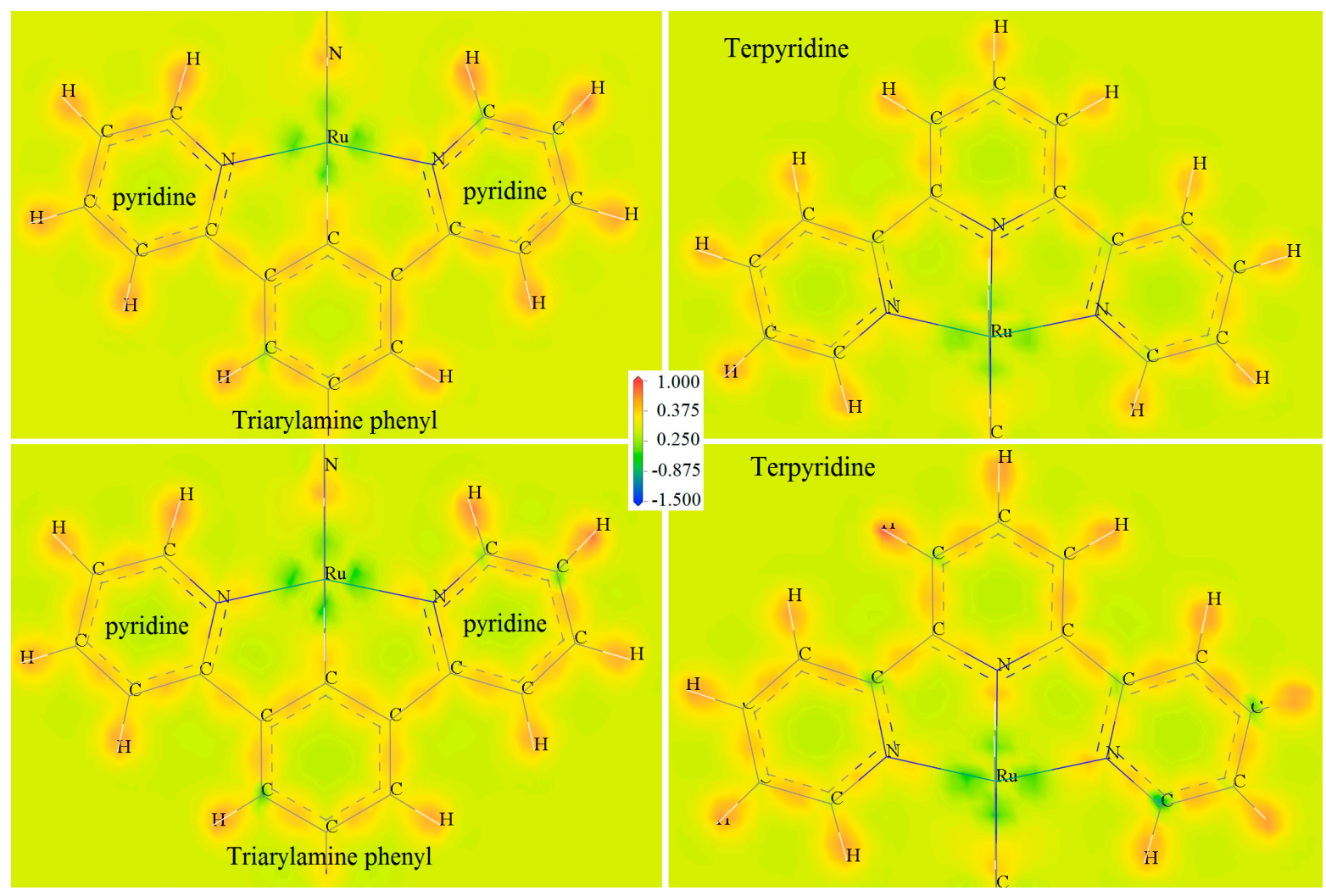

Figure 3. Deformation electron densities contoured on atom planes of triarylamine-phenyl (left panels) and terpyridine (right panels) of CTTC in +3 (top panels) and +6 (bottom panels) redox states. Atomic cores are labeled by element symbols.

Although three Ru coordination centers in CTTC molecule are almost separated in space to independently perform $\mathrm{Ru}$ (II)/(III) transition in redox reactions with three terpyridine terminals under different coordination environments, it is not reasonable to individually consider three $\mathrm{Ru}$ cations which are symmetrically conjugated by triarylamine core of CTTC molecule. Therefore, we take three Ru cations in coordination centers as a whole to identify multiple redox states of CTTC molecule by initially specifying three Ru ions to be in the same oxidation state for first-principles calculations of electronic structure and optical absorption spectrum, as mentioned in Section 2.

\subsection{Electronic Orbitals and Density of States}

The calculated electronic structures of CTTC molecular structures in different redox states obtained from geometric optimization are represented by molecular orbital energy spectra and density of electronic states (DOS), as shown in Figure 4. Energy levels of electronic states concentrate to energy bands above the lowest unoccupied molecular orbital (LUMO) and below the highest occupied molecular orbital (HOMO). CTTC molecule represents a semiconducting character of electronic energy bands, with the band-gap declining from 1.4 to $0.4 \mathrm{eV}$ when $(\mathrm{Ru})_{3}$ oxidation state (CTTC redox state) increases from +2 to +6 . Only in +4 redox state, electronic spin-polarization of CTTC leads to obvious energy spin splittings near HOMO so that a part of down-spin $(\beta)$ states is converted to up-spin $(\alpha)$ states, with the residual $\beta$-states shifting towards lower energy, merely remaining $\alpha$-state electrons at HOMO. Therefore, a higher number of up-spin states than down-spin states are 
occupied in valence band (below HOMO) to engender a net spin of CTTC molecule (molecular magnetic moment). As comparatively noted from Figure $4 \mathrm{~b}-\mathrm{d}$, with the increase in redox state, the highly degenerated energy levels of unoccupied molecular orbitals gradually split into an extended band ( +4 and +5 states) and even into uniformly distributed multiple minibands (+6 state) in the range of $1.5 \sim 3.1 \mathrm{eV}$ above $\mathrm{HOMO}$, which is completely included into visible region of optoelectronic transitions.
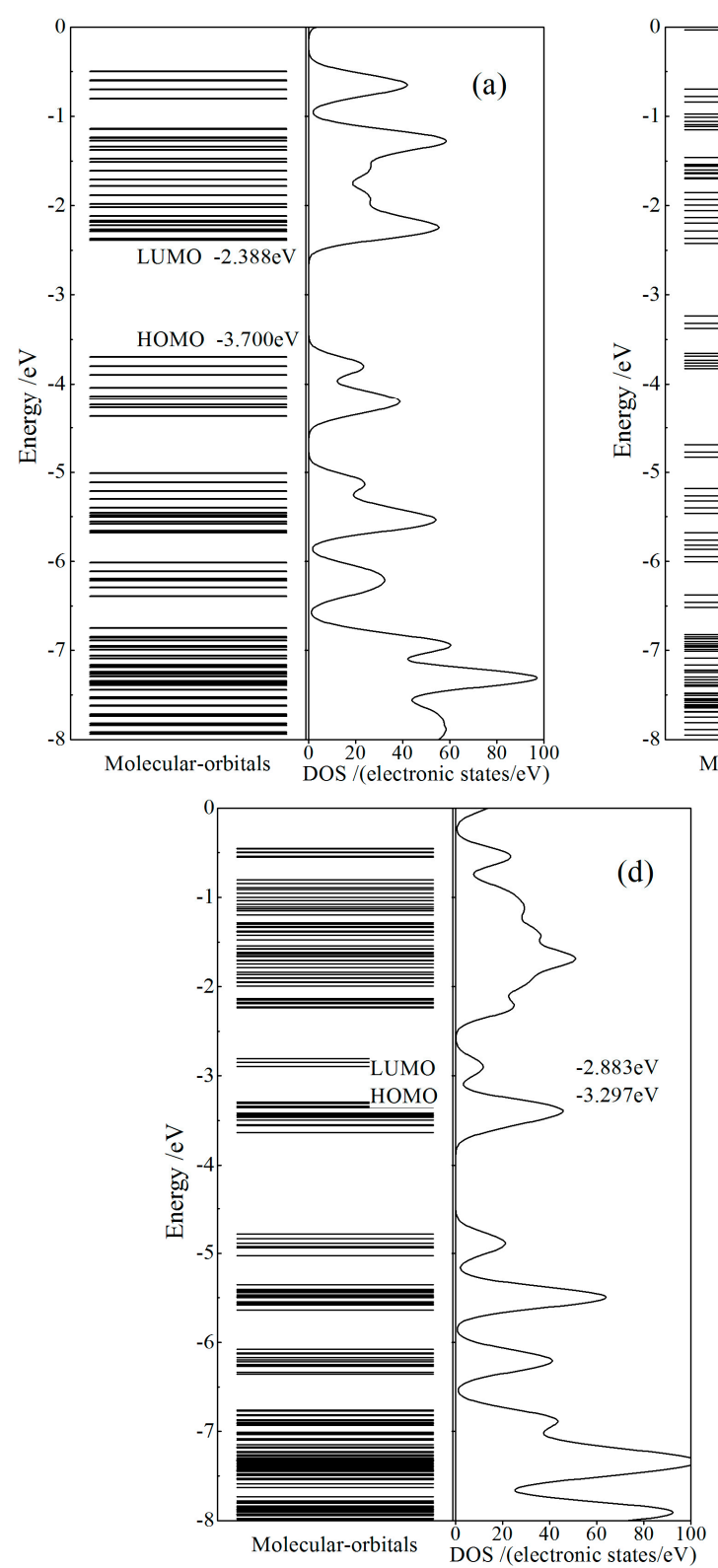

(b)

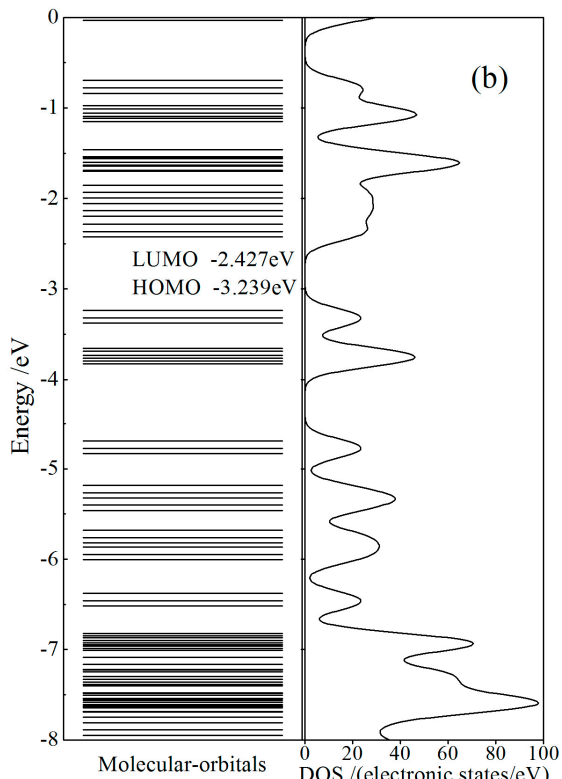

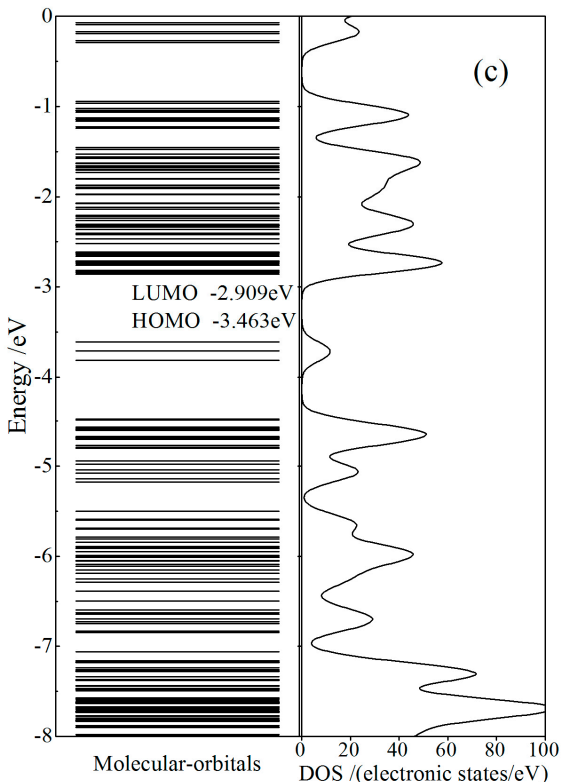

(e)

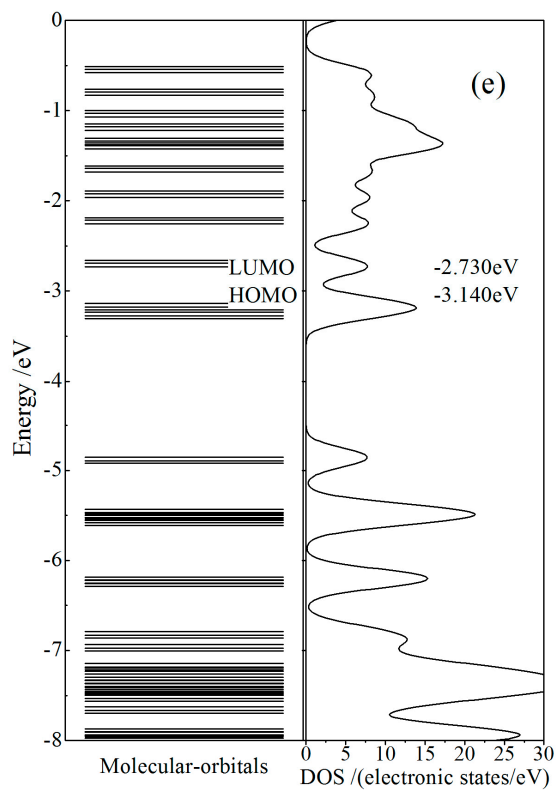

Figure 4. Molecular orbital energy spectra (left panels) and densities of states (DOS, right panels) of CTTC molecule in redox states of $(\mathbf{a})+2,(\mathbf{b})+3(\mathbf{c})+4,(\mathbf{d})+5$ and $(\mathbf{e})+6$, with vacuum free electron level being referenced as energy zero and $\mathrm{HOMO} / \mathrm{LUMO}$ being labeled.

Orbital-dependent (partial) atomic-projected density of state (PDOS) indicates orbital components and atomic contributions for intrinsic electron states of CTTC molecule, which are essential to elucidate electronic attributes in multistate redox alternations, as shown in Figure 5. Electronic states at HOMO are majorly derived from Ru $4 d$-orbitals (Ru- $4 d$ ), with a minor contribution from $2 p$-orbitals of $\mathrm{N}$ and $\mathrm{C}$ atoms (N-2p and C-2p). In contrast, LUMO only consists of the major C-2p and minor N-2p components. Due to the electron 
transfer partially from $\mathrm{Ru}$ cations to the coordinating $\mathrm{N}$ and bonding $\mathrm{C}$ atoms with the increase of redox state, the five redox states are demarcated by +4 state: for +2 and +3 states, Ru- $4 d$ component of HOMO decreases in consistence with the increment in C$2 p$ and $\mathrm{N}-2 p$ components of the occupied molecule orbitals just below HOMO; for +4 state, spin polarization splits $\mathrm{HOMO}$ band into two bands so as to decrease the density of states at HOMO; for +5 and +6 states, HOMO level has been down-shifted into the high-density band just below the original HOMO due to the aggravation of electron transfer in coordination bonds, which transforms Ru- $4 d$ component to $\mathrm{C}-2 p$ and $\mathrm{N}-2 p$ components of LUMO when redox state rises from +5 to +6 . The interaction between $\mathrm{Ru}$ and coordination ligands depends essentially on the electron transfer between $\mathrm{Ru}-4 d$ and $(\mathrm{N}-\mathrm{C})-2 p$ orbitals, which determines electronic-level distributions and momentum matrix elements of optoelectronic transitions.
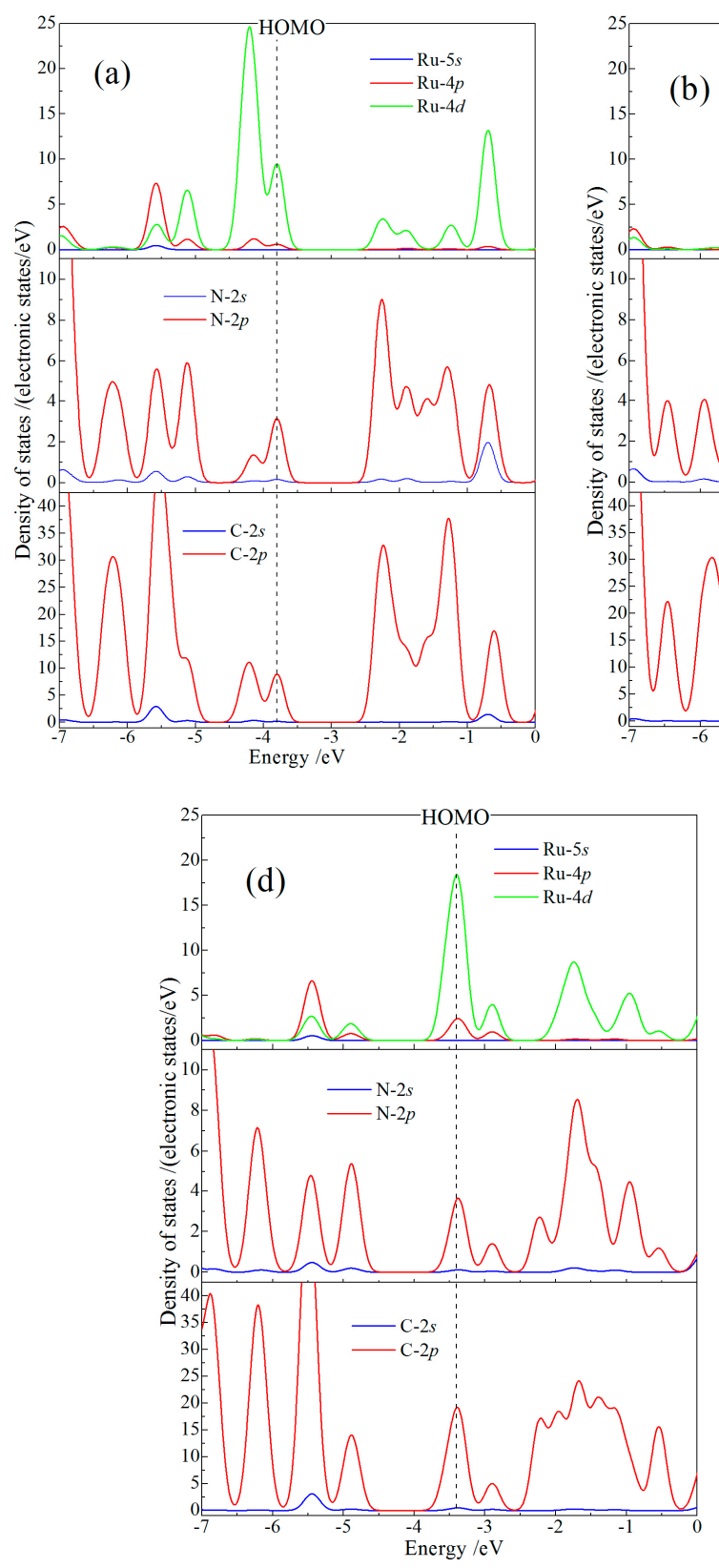
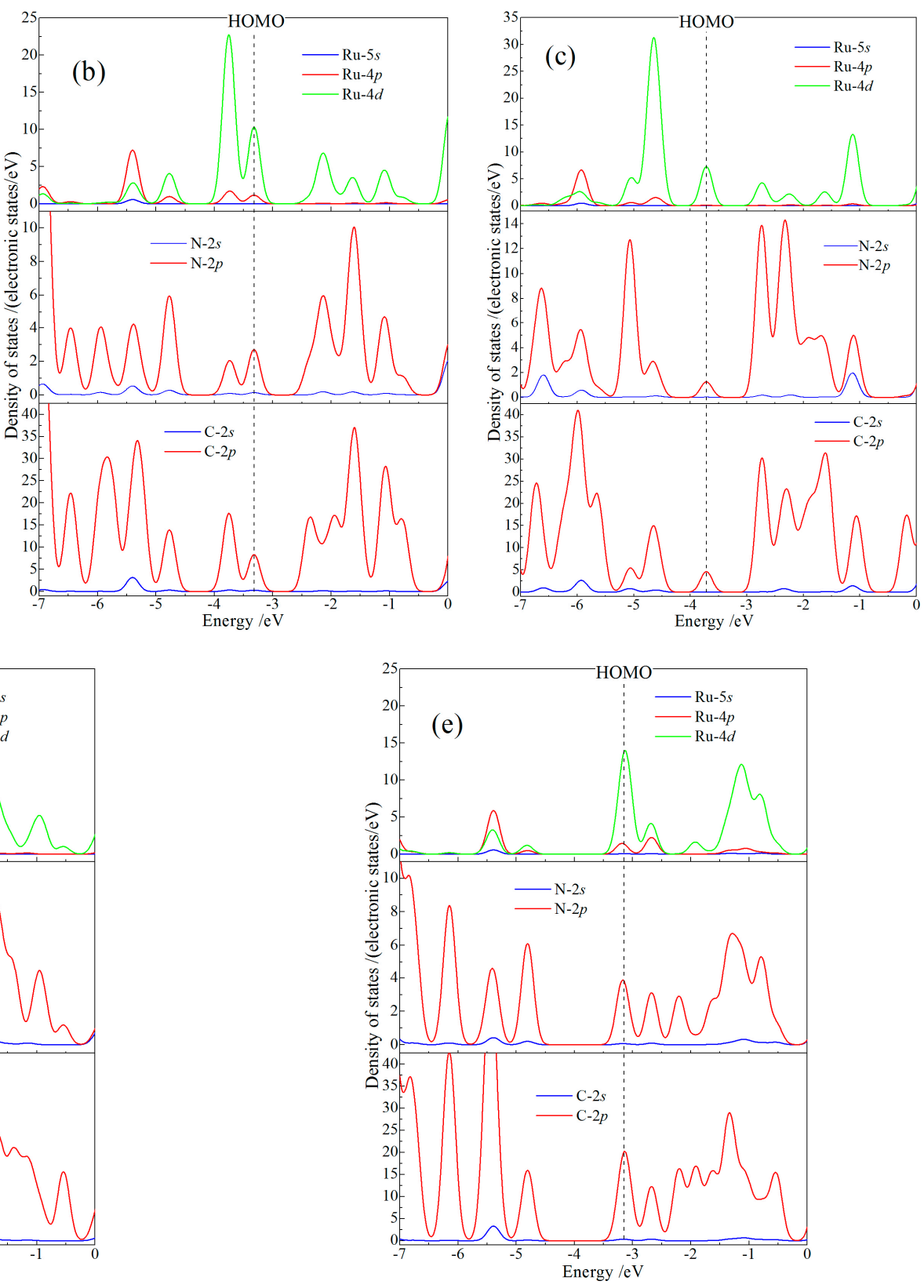

Figure 5. Total density of states and the orbital-resolved atom-projected density of states for CTTC molecule in redox states of $(\mathbf{a})+2,(\mathbf{b})+3(\mathbf{c})+4,(\mathbf{d})+5$ and $(\mathbf{e})+6$. Vacuum free electron level is referenced as energy zero. 


\subsection{Magnetic Property}

Due to ferromagnetic spin-coupling of three Ru cations at complex center, CTTC molecule presents a specific net spin of $2.25 \hbar / 2$ resulting from the sin-polarization of $\mathrm{Ru}-4 d$ orbitals only in +4 redox state. Hence, only the spin asymmetric distribution and energy splitting of +4 state CTTC is specifically analyzed to investigate magnetic attributes of CTTC, as shown in Figure 6, illustrating spin density iso-surfaces and spin-resolved partial PDOS.

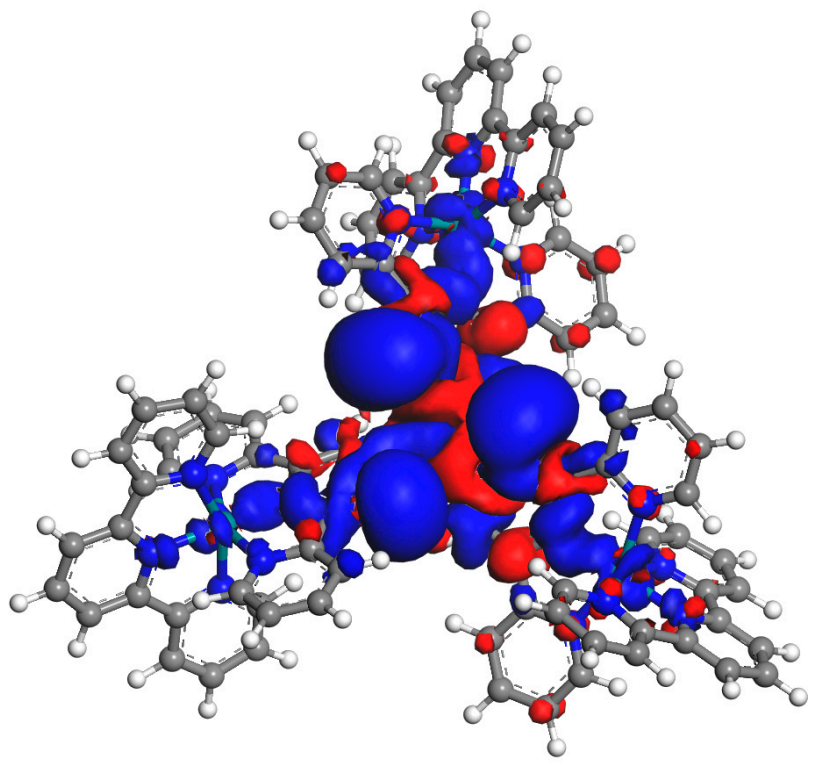

(a)

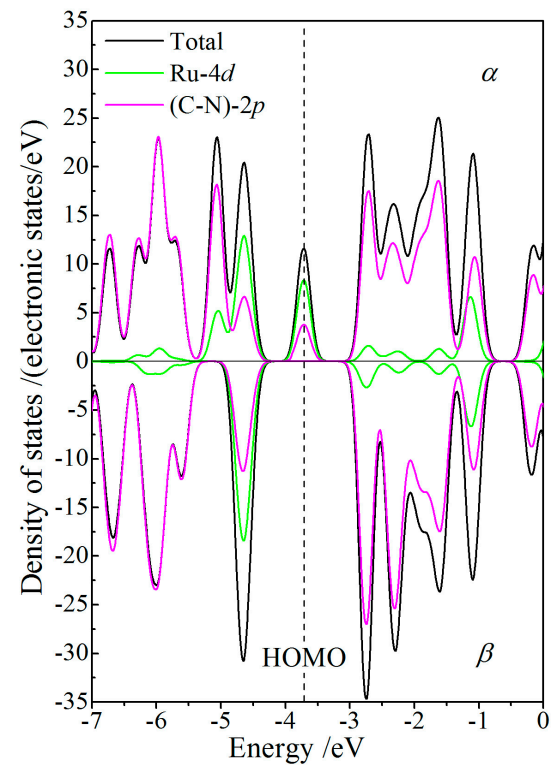

(b)

Figure 6. (a) Density iso-surfaces of spin electrons contoured at 0.005 electrons $/ \AA^{3}$ with blue and red colors indicating $\alpha$ and $\beta$ spin states, respectively; (b) spin-dependent orbital-resolved PDOS of Ru- $4 d$ and N-C- $2 p$ orbitals with vacuum free electron level being referenced as energy zero, for +4 redox state of CTTC molecule.

Spin density iso-surfaces of CTTC molecule in +4 redox state, as shown in Figure 6a, indicate the asymmetric distribution of $\alpha$ and $\beta$ states resulting from spin polarization around $\mathrm{Ru}$ cations. The major $\alpha$ and minor $\beta$ electrons are respectively distributed around the interval and internal spaces of $(\mathrm{Ru})_{3}$-conjugated triarylamine, which means the remarkably higher density of $\alpha$-state electrons than that of $\beta$-state electrons around Ru cations leads to a substantial net spin (magnetic moment). The ferromagnetic alignment of three spinpolarized Ru cations is coupling with the paramagnetic triarylamine core and diamagnetic terpyridine terminals. Meanwhile, spin polarization extends $\alpha$-states to a higher density of energy levels by shifting $\beta$-states to $\alpha$-states near HOMO in electron relaxations and thus causes the energy spin-splitting of electronic states near Fermi level, as shown in Figure 6b. Spin-splitting is partially contributed to by the ferromagnetic coupling between Ru cations, further leading to a higher number of $\alpha$-state electrons than $\beta$-state electrons. The spinand orbital-resolved PDOS of +4 state CTTC demonstrates that the energy spin-splitting of intrinsic electron states near the Fermi level is essentially derived from the spin polarization of Ru $4 d$-orbitals being coordinated by N-2p electrons and bonded with C- $2 p$ electrons, as manifested by $2 / 3 \mathrm{Ru}-4 d$ and $1 / 3$ triarylamine-(N-C)-2p components of $\alpha$-state electrons at $\mathrm{HOMO}$, further confirming that spin symmetry of $\alpha$ and $\beta$ states has been destroyed. It is thus suggested that CTTC magnetism could be under the electrical control of Ru oxidation states. 


\subsection{Optical Absorption Spectrum}

Partial transfer of coordinating electron pairs in redox reactions of electroactive CTTC can be manifested in optical absorption characteristics generated from optoelectronic transitions. As indicated by the calculated optical absorption spectra for various states of CTTC molecule in Figure 7, characteristic peaks in $24 \sim 26\left(10^{3} \mathrm{~cm}^{-1}\right)$ range originate from electron transitions of C-2p-conjugated bonds on benzene rings; optical transitions from $2 p$-electrons of $\mathrm{C}-\mathrm{N}$ conjugated bonds in pyridine and terpyridine appear in 20 24 $\left(10^{3} \mathrm{~cm}^{-1}\right)$ range; the discrete small absorption peaks at $8 \sim 13\left(10^{3} \mathrm{~cm}^{-1}\right)$ derive from the transitions of $\mathrm{Ru}-4 d$ electrons in coordination centers; in particular, optical absorption peaks at $13 \sim 20\left(10^{3} \mathrm{~cm}^{-1}\right)$ are generated from the $\mathrm{N}-2 p$ and C-2 $p$ electrons of Ru-N coordination and the $\mathrm{Ru}-\mathrm{C}$ bond around $\mathrm{Ru}$ coordination center, which are the most sensitive to redox state and well distinguished in visible region.

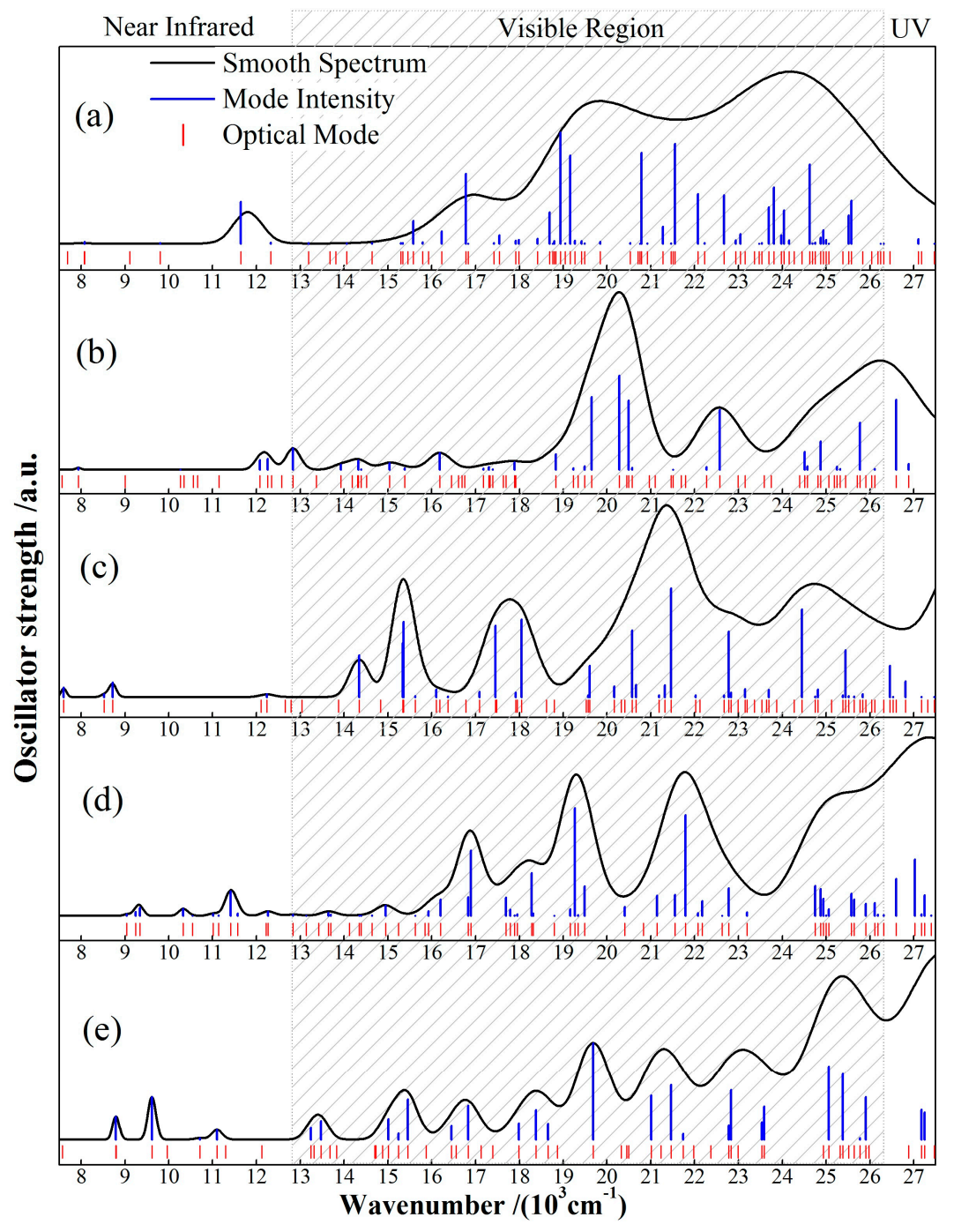

Figure 7. The calculated optical absorption spectra of CTTC in (a) $+2,(\mathbf{b})+3(\mathbf{c})+4,(\mathbf{d})+5$ and (e) +6 redox states.

CTTC complex exhibits multiple absorption peaks in wavelength range of $8 \sim 26\left(10^{3}\right.$ $\mathrm{cm}^{-1}$ ) and represents five well-identified spectra individually for five redox states, as shown in Figure 6; both +2 and +3 states show two absorption peaks in green and purple visible regions, while $+4,+5$ and +6 states present two novel absorption peaks extending from green to orange regions. As redox state rises up to +6 , CTTC complex exhibits eight characteristic absorption peaks throughout the whole visible region. In particular, 
energy spin-splitting, especially for +4 state, gives rise to orange and red absorption peaks which are attributed to electron transitions from the down-splitted Ru- $4 d$ states just below HOMO to C-2p LUMO, in contrast to yellow and orange absorption peaks of both +5 and +6 states, which derive mainly from transitions of C- $2 p$ orbitals. As listed in Table 2, the characteristic absorption wavelength, excitation energy, oscillator strength and molecular orbitals (energy levels and orbital overlap) of peak optoelectronic transitions for various redox states are sufficient to reveal multistate electrochromic performance of CTTC complex. In summary, the conjugated complexation center $(\mathrm{Ru})_{3}$ shows the red-shift and yellow-orange-red appearances of visible absorption peaks in oxidation processes from +2 to +6 states. Absorption spectrum of CTTC molecule changes from a wide green-purple band to green and purple peaks, then gives rise to orange/yellow absorption, and finally averts to a variety of uniform absorption peaks in the whole visible region.

Table 2. Absorption wavelength $\lambda_{\mathrm{m}}$, excitation energy $E_{\mathrm{m}}$ (reference to vacuum electron level), oscillator strength $O_{s}$, molecular orbital energy level and overlap in characteristic optoelectronic transitions of CTTC molecule under different redox states.

\begin{tabular}{|c|c|c|c|c|c|c|}
\hline \multirow[t]{2}{*}{ Redox State } & \multirow[t]{2}{*}{$\lambda_{\mathrm{m}} / \mathrm{nm}$} & \multirow[t]{2}{*}{$E_{\mathrm{m}} / \mathrm{eV}$} & \multirow[t]{2}{*}{$O_{\text {s }} /$ a.u. } & \multicolumn{2}{|c|}{$\begin{array}{c}\text { Molecular Orbital } \\
\text { Level/eV }\end{array}$} & \multirow[t]{2}{*}{ Orbital Overlap } \\
\hline & & & & From & To & \\
\hline+2 & 528 & 2.35 & 0.09 & -5.08 & -2.72 & 0.41 \\
\hline+3 & 493 & 2.52 & 0.29 & -3.32 & -1.09 & 0.50 \\
\hline+4 & 464 & 2.67 & 0.16 & -3.86 & -1.24 & 0.39 \\
\hline \multirow[b]{2}{*}{+5} & 519 & 2.39 & 0.27 & -3.34 & -1.84 & 0.77 \\
\hline & 459 & 2.70 & 0.25 & -3.47 & -1.00 & 0.59 \\
\hline+6 & 399 & 3.11 & 0.18 & -4.86 & -1.96 & 0.47 \\
\hline
\end{tabular}

\section{Conclusions}

Redox magnetism and electrochromism of a triangle-based cyclometalated triruthenium complex with triarylamine as a conjugated-ligand core are investigated by firstprinciples electronic structure calculations. Separated ruthenium atoms as conjugating complexation centers are one-electron-oxidized subsequently to calculate molecular net spin and optical absorption for elucidating electrochromic properties of ruthenium-complexed triphenylaminium. CTTC Complex represents a semiconductor band-gap which decreases from 1.4 to $0.4 \mathrm{eV}$ when shifting from low to high redox states for realizing multicolor electrochromic performance in visible region and possesses a considerable net spin magnetic moment in +4 redox state. The spin polarization of $\mathrm{Ru}-4 d$ orbitals coordinated by $\mathrm{N}-2 p$ and bonded with C-2p electrons leads to the molecular magnetic moment of +4 state CTTC complex, as manifested by spin-coupling of the ferromagnetic-coupled Ru cations with the paramagnetic triarylamine core and diamagnetic terpyridine terminals. Densities of electronic states also reveal optoelectronic-excitation attributes for all the five $(\mathrm{Ru})_{3}$ oxidation states that could present four consecutive couples of one-electron redox. The exceptional net spin of +4 state and the characteristic optical absorption spectrum of each distinguished redox state suggest that CTTC complex is a prospective electrochromic material for electric field detection. Particularly, significant contrasts between optical absorption wavelengths of different redox states in visible region have been theoretically demonstrated to be applied for optical inspection techniques in electrical power systems.

Author Contributions: Conceptualization, W.S. and W.-F.S.; methodology, L.L.; software, M.-Y.C. and Y.W.; investigation, Y.-X.S. and W.-F.S.; data curation, J.Z. and J.-Q.L.; writing-original draft preparation, Y.-X.S. and W.S.; writing-review and editing, W.-F.S.; funding acquisition, W.S. and H.-Q.L. All authors have read and agreed to the published version of the manuscript. 
Funding: This research was funded by National Natural Science Foundation of China (Grant No. 51607048) and partially supported by the scientific project "Detecting Flashover Failure of Composite Insulator in Cold Environment" (Grant No. SGHLDK00PJJS1900143) from State Grid Heilongjiang Electric Power Co. Ltd. of China.

Informed Consent Statement: Informed consent was obtained from all subjects involved in the study.

Data Availability Statement: Calculation methods and results are available from the authors.

Conflicts of Interest: The authors declare no conflict of interest.

\section{References}

1. Carpi, F.; Rossi, D.D. Colours from electroactive polymers: Electrochromic, electroluminescent and laser devices based on organic materials. Optic. Laser Technol. 2006, 38, 292-305. [CrossRef]

2. Cui, B.B.; Nie, H.J.; Yao, C.J.; Yao, C.J.; Shao, J.Y.; Zhong, Y.W. Reductive electropolymerization of bis-tridentate ruthenium complexes with 5,5"-divinyl-4'-tolyl-2,2':6',2"-terpyridine. Dalton Trans. 2013, 42, 14125-14133. [CrossRef] [PubMed]

3. Abidin, T.; Zhang, Q.; Wang, K.L.; Liaw, D.J. Recent advances in electrochromic polymers. Polymer 2014, 55, 5293-5304. [CrossRef]

4. Shao, J.Y.; Yao, C.J.; Cui, B.B.; Gong, Z.L.; Zhong, Y.W. Electropolymerized films of redox-active ruthenium complexes for multistate near-infrared electrochromism, ion sensing, and information storage. Chin. Chem. Lett. 2016, 27, 1105-1114. [CrossRef]

5. Tang, J.H.; Yao, C.J.; Zhong, Y.W.; Cui, B.B. Ruthenium-amine conjugated organometallic materials for multistate near-IR electrochromism and information storage. Chem. Rec. 2016, 16, 754-767. [CrossRef] [PubMed]

6. Simao, C.; Mas-Torrent, M.; Casado-Montenegro, J.; Otón, F.; Veciana, J.; Rovira, C. A three-state surface-confined molecular switch with multiple channel outputs. J. Am. Chem. Soc. 2011, 133, 13256-13259. [CrossRef]

7. Yoshida, M.; Yashiro, N.; Shitama, H.; Kobayashi, A.; Kato, M. A redox-active dinuclear platinum complex exhibiting multicolored electrochromism and luminescence. Chem. A Euro. J. 2015, 22, 491-495. [CrossRef]

8. Norel, L.; Tourbillon, C.; Warnan, J.; Pellegrin, Y.; Miomandre, F.; Odobel, F.; Rigaut, S. Redox-driven porphyrin based systems for new luminescent molecular switches. Dalton Trans. 2018, 47, 8364-8374. [CrossRef]

9. Lim, H.; Seo, S.; Pascal, S.; Bellier, Q.; Rigaut, S.; Park, C.; Shin, H.; Maury, O.; Andraud, C.; Kim, E. NIR electrofluorochromic properties of aza-boron-dipyrromethane dyes. Sci. Rep. 2016, 6, 1-8.

10. Uflyand, I.E.; Dzhardimalieva, G.I. Molecular design of supramolecular polymers with chelated units and their application as functional materials. J. Coor. Chem. 2018, 71, 1271-1356. [CrossRef]

11. Banasz, R.; Walesa-Chorab, M. Polymeric complexes of transition metal ions as electrochromic materials: Synthesis and properties. Coor. Chem. Rev. 2019, 389, 1-18. [CrossRef]

12. Mas-Torrent, M.; Rovira, C.; Veciana, J. Surface-confined electroactive molecules for multistate charge storage information. Adv. Mater. 2013, 25, 462-468. [CrossRef] [PubMed]

13. Wang, H.; Barrett, M.; Duane, B.; Gu, J.; Zenhausen, F. Materials and processing of polymer-based electrochromic devices. Mater. Sci. Eng. B 2018, 228, 167-174. [CrossRef]

14. Laschuk, N.O.; Obua, A.; Ebralidze, I.I.; Fruehwald, H.M.; Poisson, J.; Egan, J.G.; Gaspari, F.; Naumkin, F.Y.; Easton, E.B.; Zenkina, O.V. Spacer conjugation and surface support effects in monolayer electrochromic materials. ACS Appl. Electron. Mater. 2019, 1, 1705-1717. [CrossRef]

15. Yao, C.J.; Zhong, Y.W.; Nie, H.J.; Abruña, H.D.; Yao, J.N. Near-IR electrochromism in electropolymerized films of a biscyclometalated ruthenium complex bridged by 1,2,4,5-tetra(2-pyridyl)benzene. J. Am. Chem. Soc. 2011, 133, 20720-20723. [CrossRef] [PubMed]

16. Grelaud, G.; Cifuentes, M.P.; Schwich, T.; Argouarch, G.; Petrie, S.; Stranger, R.; Paul, F.; Humphrey, M.G. Multistate redox-active metalated triarylamines. Eur. J. Inorg. Chem. 2012, 145, 65-75. [CrossRef]

17. Shen, J.J.; Zhong, Y.W. Long-range ruthenium-amine electronic communication through the para-oligophenylene wire. Sci. Rep. 2015, 5, 13835. [CrossRef]

18. Zhong, Y.W.; Gong, Z.L.; Shao, J.Y.; Yao, J.N. Electronic coupling in cyclometalated ruthenium complexes. Coord. Chem. Rev. 2016, 312, 22-40. [CrossRef]

19. Qian, G.; Wang, Z.Y. Near-infrared organic compounds and emerging applications. Chem. Asian J. 2010, 5, 1006-1029. [CrossRef]

20. Polit, W.; Exner, T.; Wuttke, E.; Winter, R.F. Vinylruthenium-triarylamine conjugates as electroswitchable polyelectrochromic NIR dyes. Bioinorg. React. Mech. 2012, 8, 85-105. [CrossRef]

21. Nagashima, T.; Ozawa, H.; Suzuki, T.; Nakabayashi, T.; Kanaizuka, K.; Haga, M.A. Photoresponsive molecular memory films composed of sequentially assembled heterolayers containing ruthenium complexes. Chem. Eur. J. 2016, 22, 1658-1667. [CrossRef] [PubMed]

22. Siepmann, J.I.; Frenkel, D. Configurational bias Monte Carlo: A new sampling scheme for flexible chains. Mol. Phys. 1992, 75, 59-70. [CrossRef]

23. Tang, J.H.; He, Y.Q.; Shao, J.Y.; Gong, Z.L.; Zhong, Y.W. Multistate redox switching and near-infrared electrochromism based on a star-shaped triruthenium complex with a triarylamine core. Sci. Rep. 2016, 6, 35253. [CrossRef] [PubMed] 
24. Delley, B. From molecules to solids with the DMol3 approach. J. Chem. Phys. 2000, 113, 7756-7764. [CrossRef]

25. Andzelm, J.; King-smith, R.D.; Fitzgerald, G. Geometry optimization of solids using delocalized internal coordinates. Chem. Phys. Lett. 2001, 335, 321-326. [CrossRef]

26. Perdew, J.P.; Ruzsinszky, A.; Csonka, G.I.; Vydrov, O.A.; Scuseria, G.E.; Constantin, L.A.; Zhou, X.; Burke, K. Restoring the density-gradient expansion for exchange in solids and surfaces. Phys. Rev. Lett. 2008, 100, 136406. [CrossRef]

27. Chantis, A.N.; Christensen, N.E.; Svane, A.; Cardona, M. Full-zone analysis of relativistic spin splitting at band anticrossings: The case of zinc-blende semiconductors. Phys. Rev. B 2010, 81, 205205. [CrossRef]

28. Kresse, G.; Furthmüller, J. Efficient iterative schemes for ab initio total-energy calculations using a plane-wave basis set. Phys. Rev. $B$ 1996, 54, 11169. [CrossRef]

29. Baker, J.; Kessi, A.; Delley, B. The generation and use of delocalized internal coordinates in geometry optimization. J. Chem. Phys. 1996, 105, 192-212. [CrossRef]

30. Tkatchenko, A.; Scheffler, M. Accurate molecular van der Waals interactions from ground-state electron density and free-atom reference data. Phys. Rev. Lett. 2009, 102, 073005. [CrossRef]

31. Delley, B. Time dependent density functional theory with DMol3. J. Phys. D Condens. Matter. 2010, 22, 384208. [CrossRef] [PubMed] 